\title{
Effectiveness of Closure of Public Places with Time Delay in Disease Control
}

\author{
Zhenggang Wang ${ }^{1,2,3}$, Kwok Yip Szeto ${ }^{1}$ and Frederick Chi-Ching Leung ${ }^{2}$ \\ ${ }^{1}$ Department of Physics, Hong Kong University of Science and Technology, Clear Water Bay, Hong \\ Kong SAR, People's Republic of China \\ ${ }^{2}$ Department of Zoology, The University of Hong Kong, Pokfulam Road, Hong Kong SAR, People's \\ Republic of China
}

\begin{abstract}
Summary
A theoretical basis for the evaluation of the efficiency of quarantine measure is developed in a SIR model with time delay. In this model, the effectiveness of the closure of public places such as schools in disease control, modeled as a high degree node in a social network, is evaluated by considering the effect of the time delay in the identification of the infected. In the context of the SIR model, the relation between the number of infectious individuals who are identified with time delay and then quarantined and those who are not identified and continue spreading the virus are investigated numerically. The social network for the simulation is modeled by a scale free network. Closure measures are applied to those infected nodes with high degrees. The effectiveness of the measure can be controlled by the present value of the critical degree $K_{C}$ : only those nodes with degree higher than $K_{C}$ will be quarantined. The cost $C_{Q}$ incurred for the closure measure is assumed to be proportional to the total links rendered inactive as a result of the measure, and generally decreases with $K_{C}$, while the medical cost $C_{Q}$ incurred for virus spreading increases with $K_{C}$. The total social $\operatorname{cost}\left(C_{M}+C_{Q}\right)$ will have a minimum at a critical $K^{*}$, which depends on the ratio of medical cost coefficient $\alpha_{M}$ and closure cost coefficient $\alpha_{Q}$. Our simulation results demonstrate a mathematical procedure to evaluate the efficiency of quarantine measure. Although the numerical work is based on a scale free network, the procedure can be readily generalized and applied to a more realistic social network to determine the proper closure measure in future epidemics.
\end{abstract}

\section{Introduction}

The recent outbreaks of highly pathogenic avian influenza (H5N1) in Southeast Asia, Europe and Africa [1] not only caused great loss in poultry, but also poses imminent pandemic threat to humans $[2,3]$. To prevent similar disaster from occurring, several non-pharmaceutical interventions were proposed to mitigate the losses due to the pandemics $[4,5]$. These measures are aimed at decreasing the probability of exposure of the susceptible to the infected, by social distancing and avoidance of crowding [6].

In a social network, places such as housing projects, schools, public transit, factories and offices, all play an important role in virus transmission [7] as they are hubs of peoples' daily activities [8]. A good example is the outbreak of SARS in Hong Kong. The first infected case,

\footnotetext{
${ }^{3}$ To whom correspondence should be addressed. E-mail: phzgwang@ust.hk
} 
who is an old professor, stayed in a hotel and infected seven guests who had stayed on the same floor. One of these hotel guests was responsible for the outbreak in a public hospital affecting at least 88 healthcare workers and 18 medical students. Meanwhile, a patient discharged from the same ward of the public hospital caused another outbreak affecting 237 residents in a housing estate [9]. Later, the SARS virus was transmitted overseas by international flight.

This tragedy suggests that these public places should be considered as the basic units in the practice of prevention and interventions, such as disinfection, closure and travel constraint. Specifically, the closure of public places with high connectivity, such as schools, is necessary to cut down the social contact that will spread the disease in the initial stage of outbreak [4]. Once an outbreak occurs, people are recommended to avoid crowding conscientiously and to practice good personal hygiene, such as wearing facemasks in public, disinfection and washing hands. If the outbreak is local, travel constraints are practiced so that entering and exiting an infected area is limited [4]. The practice of these non-pharmaceutical interventions necessarily changes the social activities and individual behaviors, and may cause social disruption and destruction, as reported during the outbreak of SARS in Hong Kong [10, 11]. If one models the centers of social activities as nodes in a social network, the seriousness of the non-pharmaceutical intervention can be mathematically described by the closure of these centers, where social activities associated with them are measure by the degree of these nodes. For example, the closure of public places like school can be a very effective measure to reduce the risk of virus spreading among students.

In the decision making process by the administration with the authority to close public places, the selection process can be based on the popularity or connectedness of these public places. In a social network, this can be described by nodes with degree above some chosen value $K_{C}$. We see that the larger the $K_{C}$, the less social places are affected for there are few such nodes in a social network. Also, the choice of nodes for closure depends on how pathogenic is the virus and medical treatment available.

While the closure of centers of social activities is effective in the prevention of virus spreading via social contact, the cost will be high if an unreasonable amount of activities are interrupted. On the other hand, if too few high degree nodes are quarantined, the number of infected people will only increase and the medical cost, such as hospitalization, will also increase. Therefore, there must be a balance between the cost for closure and the cost associated with medical treatment for the infected. Our aim is to use the SIR model with time delay to numerically obtain the optimal measure that minimize the total cost, assuming a fixed ratio of the cost for closure and cost for medical treatment. The cost of medical treatment (including the economic and physical losses of the patient due to infection, even the cost of life) is to be estimated at the initial stage of the virus outbreak, co-determined by the pathogenicity of the virus and the availability of the effective medical treatment. The cost of closure can be estimated based on investigation, for instance, in the case of the closure of a shopping mall, the loss of business is a main contributor of economic loss. In our simple model, we assume that the cost of closure of a node is proportional to the daily activity taking place in that node. Furthermore, a measure of these activities is approximated by the degree of the node. 


\section{Methods}

Recent studies of social networks reveal a special characteristic of these networks called the scale-free property, which states that the degree distribution function is a power law, $P(K) \sim$ $K^{-\gamma}, 2 \leq \gamma \leq 3$ [12]. We therefore use a scale free network to build the social network for the estimation of the influence of the practice of these measures, before discussing their efficiency and cost. Our choice of scale free network is based on the Barabasi-Albert model [13, 12], with model parameters $m=m_{0}=4$, resulting in a network with average degree $\langle K\rangle=2 m=8$ and a scale free degree distribution with $\gamma=3$. We choose these model parameters $\left(m, m_{0}\right.$ and $\left.\langle K\rangle\right)$ for convenience of illustration. These parameters are typical of many realistic networks [13, 12], while our conclusion is quite general as we aim at demonstrating the procedure of analysis rather than performing a specific case study of past data. In this network, the links between nodes describe social activities. Fig. 1 illustrates an example of nodes as homes, schools and transit centers.

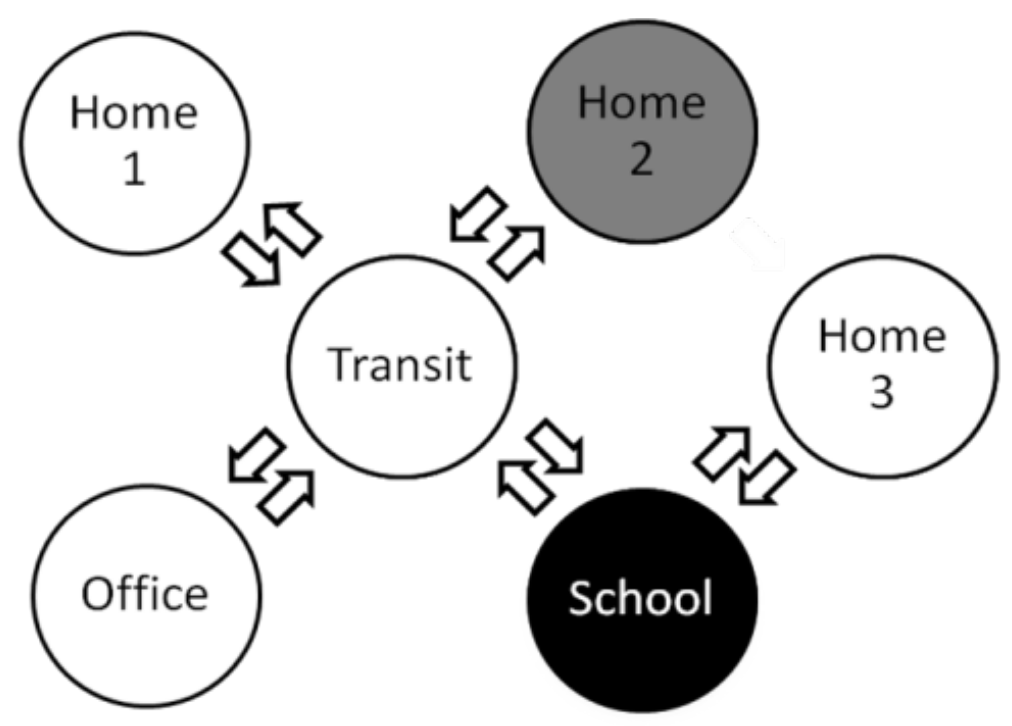

Figure 1: A schematics showing the social network made with nodes denoting center of social activities, such as schools, offices, public transit and households connected by human daily activities. In emergency, the closures of places with high connectivity, such as schools, are necessary. Schools (black circle) can be fully closed, and those affected places, where infected cases are identified, like home 2 (grey circle) should be under surveillance and control (we denote the control strength as $\delta)$.

In an emergency response to severe pandemic influenza outbreak, the government will close places with high connectivity, such as schools and public gatherings. In the context of our scale free network model for social activities, we describe the closure measure by saying that a node will be isolated if its degree $K$ is greater than a chosen critical value $K_{C}$. Consequently, the network is changed and links to these high degree nodes are disconnected. Note also that with a higher critical value $K_{C}$, the probability that a node will be isolated is less, since our degree distribution for the network is described by a power law decay. In this sense, the choice of the critical value $K_{C}$ provides a mathematical parameter in tuning the strength of the control measure. In Fig. 2a, we show the degree distribution of the network after the execution of the closure measure, where the strong measure $\left(K_{C}=15\right)$ is compared with a weaker one $K_{C}=25$, where less nodes are closed. 
In order to evaluate the effect of the administration of closure of nodes, we introduce a simple model for the cost estimation. The cost for a closed node is assumed to be proportional to the daily social activity associated with the node. Here the social activity associated with the node is assumed to be measured by its degree $K$. Now we define $\alpha_{m}$ to be medical cost per node (the cost for the medical treatment of the infected individuals), with in mind that the administration enforces the closure of the place (node) once an infected individual associated with the place is identified and hospitalized. This in fact is the strategy employed in dealing with the avian flu. We also define $\alpha_{Q}$ to be the closure cost per link for the node. Since the degree $K$ of the node is a measure of the social activities of the node, therefore the closure of this node will incur a medical cost of $\alpha_{M}$ and the social cost of closure incurred is $\alpha_{Q} K$. We further assume that $\alpha_{M}$ and $\alpha_{Q}$ are constant, which is a simplification as different people and different type of nodes have very different cost, (such as a node corresponding a shopping mall is very different from a node corresponding a school as the link connecting a school involves mostly students in the population). However, as our model is a first attempt to address the issue of the efficiency of closure measure in a mathematical approach, we cannot provide a very detailed description of the cost of different people and different types of nodes. Indeed, when closure measure is applied to a node, losses will be incurred in many forms, such as the economic loss, social loss such as the loss of class time in school, etc. In general, we expect that the closure of a place with more daily social activities, such as big shopping mall and university, will generate more losses. To model the $\operatorname{cost} C_{Q}$ for the closure of high degree nodes, we assume the cost of closing one place is proportional to its activities (the degree), thus that the total $\operatorname{cost} C_{Q}$ is proportional to the total links deleted,

$$
C_{Q}=\alpha_{Q} \sum_{K=K_{C}}^{+\infty} P(K) K
$$

The parameter $\alpha_{Q}$ is the loss due to cancelling the travel to some public places (for instance, student missing classes). In our simple model, we assume $\alpha_{Q}$ to be a constant. When we apply a strong measure, $K_{C}$ is small, the cost is high, as illustrated in Fig. $2 \mathrm{~b}$.

A standard model to describe the dynamics of the spreading of diseases is the SIR model. Here we also adopt this model as a basis for the analysis of our control measure applied to a social network. The SIR model has been used successfully for many infectious diseases including measles, mumps and rubella. The SIR model for epidemiology was originally proposed by Kermack and McKendrick [14]. The main assumption of this model is that once a virus is introduced in a population, there are three subgroups, the susceptible (S), the infected (I) and the recovered (R) from the infection cycle, in which the three subgroups can be mutually convertible as long as the virus sustains. The three letters, S, I, and R, also represent the number of people in each subgroup at a particular time. The evolution of these numbers is governed by a differential equation with origin in population dynamics. For a specific disease in a specific population, these numbers may be worked out in order to predict possible outbreaks and bring them under control. The detailed mathematical description of this model can be found in [15].

Our model is a modified SIR model based on one key element, which is the time delay in the identification of the infected subgroup. Furthermore, our numerical simulation is performed for this SIR model with time delay on a social network. For a particular node with at least one infected individual, this person has a finite probability in transmitting the virus to others in the same location or different location via social contact, since people can move from one node to 


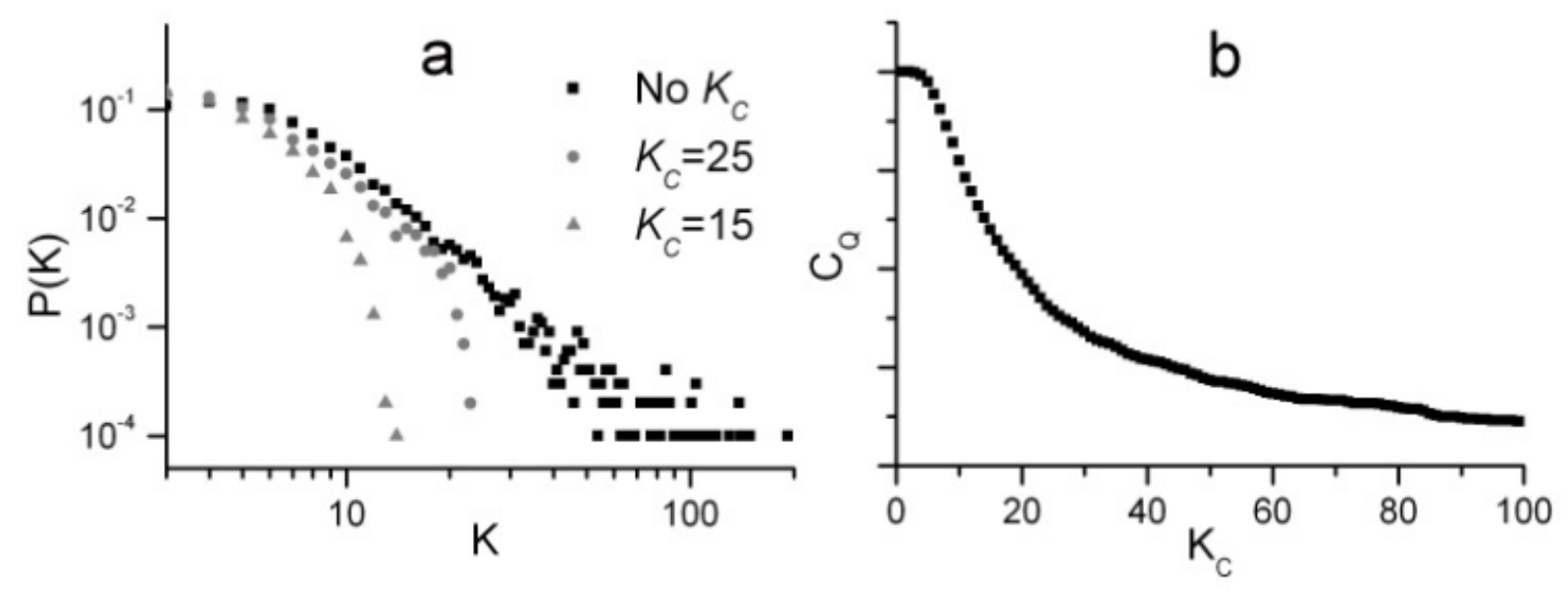

Figure 2: (a) The degree distribution of the network $P(K)$ for a given $K_{C}=15$ and 25. (b) A small $K_{C}$ presents highly strengthened measures being practiced, so that social activity is depressed and great economic losses $C_{Q}$ will be involved.

the other. The social activities between places form links in our network of places. Note that different person has different response to the virus. Some will induce severe symptom, some will recover in days with mild symptom and some will even have no symptom but are infectious for several days [11]. In our model, we take these into account by a time delay parameter. We assume that if an infected individual stays in one place, he/she will cause either mild outbreak (meaning that people here get infected and recovered quickly, but without severe symptoms), or severe outbreak (some people who are very sick are sent to hospital and the place needs to be quarantined with possible travel restriction). These complex situations are summarized in our SIR model with a single parameter $T_{I}$. For typical epidemics such as influenza, the infectious period $T_{I}$ ranges between two to seven days $[2,16]$. We can model the distribution of the infectious period $T_{I}$ by the Poisson distribution, with average four days,

$$
p\left(T_{I}\right)=\frac{e^{-a} a^{T_{I}}}{T_{I} !} \quad \text { with } \quad\left\langle T_{I}\right\rangle=\sum_{T=0}^{+\infty} T p(T)=a=4
$$

The infected individuals spread the virus and eventually recovered. However, a considerable number of the infected individuals with severe symptoms require hospitalization. Here we assume that the time delay before hospitalization is $T_{H}$, which also follows Poisson distribution with average time 4 days,

$$
p\left(T_{H}\right)=\frac{e^{-a} a^{T_{H}}}{T_{H} !} \quad \text { with } \quad\left\langle T_{H}\right\rangle=\sum_{T=0}^{+\infty} T p(T)=a=4
$$

Once a hospitalization case is identified, the place where the case is found will be considered as under control. The control of the place may include measures such as isolation, mass disinfection, and travel restriction $[17,18,19,20,21]$. In our simple model, we introduce a parameter called the strength of control, $(0 \leq \delta \leq 1)$, which represents the efficiency of the control of the place in the prevention of the spreading of the virus to other places. Here we normalize this parameter so that a fully closed place corresponds to $\delta=1$ and a place without control measure corresponds to $\delta=0$. 
We perform numerical simulation of outbreak of pandemic influenza using the scale free network and the SIR model with time delay with control measure parameter $\delta$. The cost of hospitalization and control measure applied to a node, is modeled by $C_{M}=\alpha_{M} N_{M}$, where $N_{M}$ is the number of places where infected cases are found. Even though the mathematical solution for coupled differential equation with time delay on a network is very difficult, the numerical simulation is relatively easy. We next discuss the results of our numerical work.

\section{Results}

Our simulation results indicate that there are two key parameters that control the loss incurred by the pandemic. The first parameter is $K_{C}$. A small value of $K_{C}$ implies the closure of many places for social activities, thereby containing the outbreak efficiently. The number of cases for hospitalization, $N_{M}$, is small and the medical cost is low, as shown in Fig. 3a. However, the social cost due to reduced economic and social activities is high, as shown in Fig. 2 b.
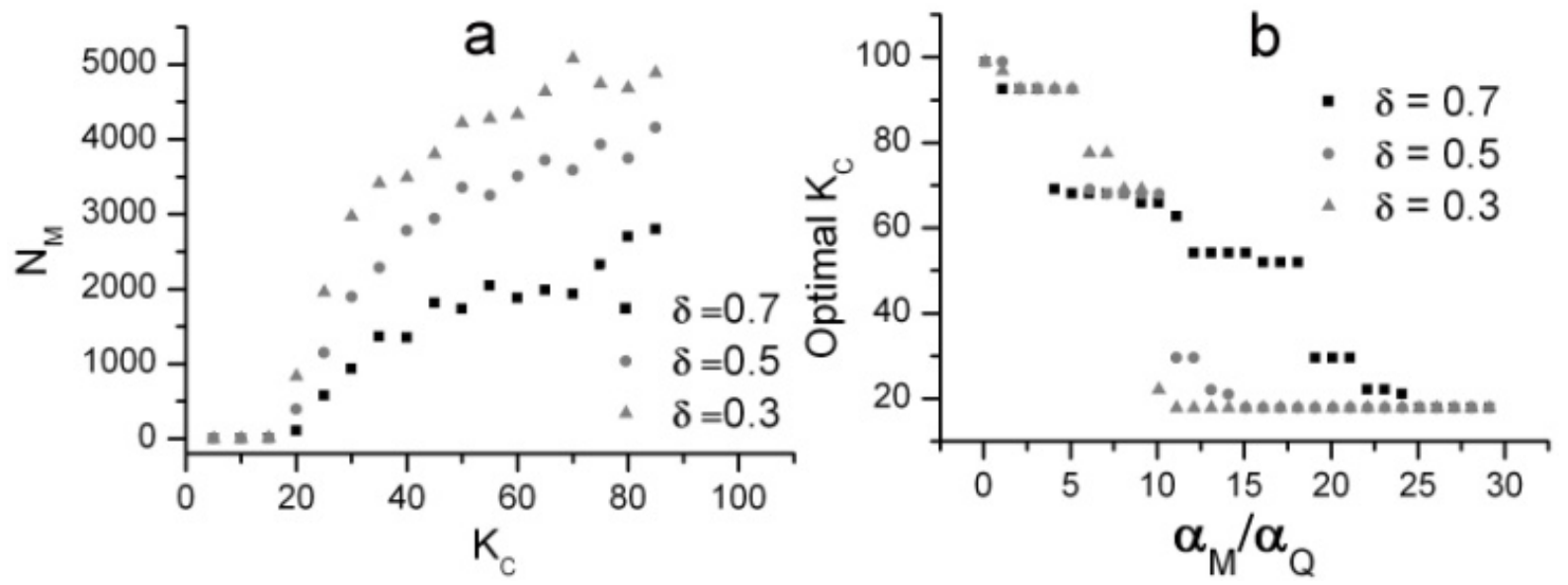

Figure 3: (a) The counted total number of places under control in the first 200 days since the outbreak, with different $K_{C}$ and $\delta$. (b) The optimal $K_{C}$, which minimizes total cost for containing the pandemic influenza outbreak $C_{M}+C_{Q}$, with different $\alpha_{M} / \alpha_{Q}$

The second key parameter is the strength of the control measure $\delta$ applied to the places where infected cases with serious symptoms are found. A high value $\delta$ implies strict and effective closure of the affected area and the hospitalization cost is low due to low value of $N_{M}$. On the other hand, a low value of $\delta$ means that the control measure is not very strict, and the medical cost incurred is high. This again is clearly shown in Fig. 3a.

Since the cost for interventions $C_{Q}$ monotonically decreases with $K_{C}$, while the medical cost $C_{M}$ monotonically increases with $K_{C}$, we expect an optimal value $K^{*}$ of $K_{C}$ can be found to minimize the total cost $\left(C_{M}+C_{Q}\right)$. Since the costs depends on the medical cost $\alpha_{M}$ and closure cost $\alpha_{Q}$ per person, the optimal value $K^{*}$ will be dependent on the ratio $\alpha_{M} / \alpha_{Q}$, as well as the strength of control measure $\delta$. This is shown in Fig. $3 \mathrm{~b}$.

Based on a known ratio of $\alpha_{M} / \alpha_{Q}$ in a given social environment, and the level of execution of the control measure $\delta$ for the given administration, one can decide the optimal value $K^{*}$ that will minimize the total cost. The procedure is based on numerical simulation of the SIR model with time delay. 


\section{Discussion}

One of the many simplifying assumptions in our model for measuring the effectiveness of the closure of public places with time delay in disease control concerns the estimation of the various parameters. Specifically, we encounter many difficulties in the estimation of $\alpha_{M}$ and $\alpha_{Q}$, we should note that for an outbreak associated with a new virus, our estimates of these cost parameters rely heavily on our limited knowledge of the new virus, in addition to the unpredictable consequences of the practice of the control measures and the inexperience in the execution of the control measures since the virus is new. As one may expect, these uncertainties will introduce errors and affect the administrative decision. However, our model and analysis do provide a methodology of handling these complex situations. Indeed, our analysis demonstrates the importance of the accurate estimation of the effect of the new virus and the value of efficient and timely execution of control measure at the INITIAL stage of the outbreak. It is our aim to point out the method of analysis that leads to a scientifically sound approach to the administration of control measure, rather than performing a specific case studies using past data.

A second item worthy for further research concerns the modification of the social network under control measure. Of course, the removal or closure of the high degree nodes will change the structure of the network. The social network after the administration measure will likely to be different from the original one with a power-law degree distribution. This is quite reasonable and understandable, as any major decision on closure of high degree nodes will affect social behavior dramatically. Closure of a shopping mall has a very different effect from the closure of a school, for example. However, within the confine our limited knowledge of the social network under an outbreak and the associated decisions on closure of different types of high degree nodes, our model can only address this issue of changing social network by saying that we treat the modification on the social network after the first round of administrative decision on control measure as a second order perturbation and our conclusion hopefully will not be greatly changed by this second order effect. After all, we must emphasize the tremendous impact of the control measure on social society. As shown in Fig. 2a, the execution of control measure affect the social behavior dramatically, as social activities greatly decreases and the public places are out of service. Although the closure of public places is proven to be an efficient measure in controlling the spreading of virus, the cost incurred to the entire society is huge. Therefore, it is important to provide a quantitative attempt to evaluate the timely and practical control measure, such as the closure of public places, in the fight against the spreading of new virus.

In summary, we have presented a simple model using several approximations to estimate the optimal measure for closure of social activities under the threat of pandemic that would minimize the total cost, which include cost of disruption of economic and social activities and medical cost of treatment of the infected. The approximations include using a scale free network to model the social network, a Poisson distribution for the time delays, and the standard SIR model defined on a network of places of social activities. The procedure used in our model however can be readily generalized to more complex situations, where more cost factors are involved. The important thing to emphasize is that we provide a theoretical framework to estimate the different control measures that can be executed and the procedures to compute via simulation the optimal measure that minimize the total cost. 


\section{Acknowledgements}

K.Y. Szeto acknowledges support by grants CIDHHS04/05.SC02 and CERG603203.

\section{References}

[1] R. G. Webster, M. Peiris, H. Chen, and Y. Guan. H5N1 outbreaks and enzootic Influenza. Emerging Infectious Diseases, 12(1):3-8, 2006.

[2] G. Brankston, L. Gitterman, Z. Hirji, C. Lemieux, and M. Gardam. Transmission of Influenza a in human beings. The Lancet Infectious Diseases, 7(4):257-265, 2007.

[3] N. M. Ferguson, C. Fraser, C. A. Donnelly, A. C. Ghani, and R. M. Anderson. Public health. public health risk from the avian H5N1 Influenza epidemic. Science, 304(5673):968-969, 2004.

[4] H. Markel, H. B. Lipman, J. A. Navarro, A. Sloan, J. R. Michalsen, A. M. Stern, and M. S. Cetron. Nonpharmaceutical interventions implemented by US cities during the 1918-1919 Influenza pandemic. JAMA, 298(6):644-654, 2007.

[5] A. S. Monto. Global burden of Influenza: what we know and what we need to know. International Congress Series: Proceedings of the International Conference on Options for the Control of Influenza V, 1263:3-11, 2004.

[6] C. DiGiovanni, N. Bowen, M. Ginsberg, and G. Giles. Quarantine stressing voluntary compliance. Emerging Infectious Diseases, 11(11):1778-1779, 2005.

[7] D. M. Skowronski, C. Astell, R. C. Brunham, D. E. Low, M. Petric, R. L. Roper, P. J. Talbot, T. Tam, and L. Babiuk. Severe Acute Respiratory Syndrome (SARS): A year in review. Annual Review of Medicine, 56:357-381, 2005.

[8] N. J. Cox and K. Subbarao. Global epidemiology of Influenza: past and present. Annual Review of Medicine, 51:407-421, 2000.

[9] M. Chan-Yeung and W. C. Yu. Outbreak of Severe Acute Respiratory Syndrome in Hong Kong special administrative region: Case report. BMJ, 326(7394):850-852, 2003.

[10] A. S. Abdullah, B. Tomlinson, C. S. Cockram, and G. N. Thomas. Lessons from the Severe Acute Respiratory Syndrome outbreak in Hong Kong. Emerging Infectious Diseases, 9(9):1042-1045, 2003.

[11] J. K. Taubenberger, A. H. Reid, T. A. Janczewski, and T. G. Fanning. Integrating historical, clinical and molecular genetic data in order to explain the origin and virulence of the 1918 spanish Influenza virus. Philosophical Transactions of the Royal Society B: Biological Sciences, 356(1416):1829-1839, 2001.

[12] A. L. Barabasi and R. Albert. Emergence of scaling in random networks. Science, 286(5439):509-512, 1999. 
[13] R. Albert and A. L. Barabasi. Statistical mechanics of complex networks. Reviews of Modern Physics, 74(1):47-97, 2002.

[14] W. O. Kermack and A. G. McKendrick. A contribution to the mathematical theory of epidemics. Proceedings of the Royal Society of Edinburgh. Series A: Mathematics, 115(772):700-721, 1927.

[15] R. M. Anderson and R. M. May. Infectious Diseases of Humans. Oxford University Press, 1992.

[16] R. M. Anderson and R. M. May, editors. Population Biology of Infectious Disease: Report of the Workshop on Population Biology of Infectious Disease Agents. Springer, 1982.

[17] N. M. Ferguson, D. A. Cummings, S. Cauchemez, C. Fraser, S. Riley, A. Meeyai, S. Iamsirithaworn, and D. S. Burke. Strategies for containing an emerging Influenza pandemic in southeast Asia. Nature, 437(7056):209-214, 2005.

[18] N. M. Ferguson, D. A. Cummings, C. Fraser, J. C. Cajka, P. C. Cooley, and D. S. Burke. Strategies for mitigating an Influenza pandemic. Nature, 442(7101):448-452, 2006.

[19] L. O. Gostin. Influenza pandemic preparedness: Legal and ethical dimensions. Hastings Center Report, 34(5):10-11, 2004.

[20] L. O. Gostin, R. Bayer, and A. L. Fairchild. Ethical and legal challenges posed by Severe Acute Respiratory Syndrome: Implications for the control of severe infectious disease threats. JAMA, 290(24):3229-3237, 2003.

[21] L. O. Gostin, S. D. Gravely, S. Shakman, and H. Markel. Quarantine: Voluntary or not? The Journal of Law, Medicine E Ethics, 32(s4):83-86, 2004.

[22] Nicole M. Smith, Joseph S. Bresee, David K. Shay, Timothy M. Uyeki, Nancy J. Cox, and Raymond A. Strikas. Prevention and control of Influenza. recommendations of the Advisory Committee on Immunization Practices (ACIP). Morbidity and Mortality Weekly Report, 55(RR10):1-42, 2006. 